\title{
Quasiparticle scattering and local density of states in graphite
}

\author{
Cristina Bena ${ }^{1}$ and Steven A. Kivelson ${ }^{2}$ \\ ${ }^{1}$ Department of Physics, University of California, Santa Barbara, CA 93106, USA \\ ${ }^{2}$ Department of Physics and Astronomy, University of California, Los Angeles, California 90095-1547, USA
}

(Dated: August 30, 2018)

\begin{abstract}
We determine the effect of quasiparticle interference on the spatial variations of the local density of states (LDOS) in graphite in the neighborhood of an isolated impurity. A number of characteristic behaviors of interference are identified in the Fourier transformed spectrum. A comparison between our results and scanning tunneling microscopy (STM) experiments could provide a critical test of the range (of energy) of applicability of the Fermi liquid description of graphite, where some evidence of the breakdown of Fermi liquid theory has recently been discussed. Moreover, given the similarity between the band structures of graphite and that of nodal quasiparticles in a d-wave superconductor, a comparison between results in the two materials is useful for understanding the physics of the cuprates.

PACS numbers: 68.37.Ef, 81.05.Uw, 71.10.Ca
\end{abstract}

There has been considerable interest recently in Fourier transform scanning tunneling spectroscopy (FTSTS) measurements, which may reveal some rather interesting electronic properties of conducting materials. In particular, recent experiments performed on certain cuprate high $T_{c}$ superconductors $1,2,3,4,5.6 .7$, promise to shine some light on the low energy electronic excitations of the superconducting state, and on the pseudogap.

In this paper we study a simple two dimensional system, graphite, which is generally believed to be a Fermi liquid. (Note, however, that this belief has been questioned in Refs. 8.) Graphite has many similarities to the cuprates; its band structure consists of two bands that touch only at the six corners of the Brillouin zone (BZ). As a result, graphite has nodal linear dispersing quasiparticle excitations - analogous to those of the Dirac equation. This makes it in some ways analogous to a d-wave superconductor, where the nodal quasi particles are expected to have a (highly anisotropic) Dirac spectrum. Thus, our interest is in obtaining an experimentally testable understanding of the implications of quasiparticle interference, both as a test of the range of validity of Fermi liquid theory in graphite, and to provide a fiduciary point for discussions of the more complex situation in the cuprates.

We use a $T$-matrix approach ${ }^{9.10 .11}$ to obtain the quasiparticle interference spectra for various energies, in the presence of a single impurity. Our predictions can be easily tested experimentally, as very clean graphite samples are relatively easy to obtain. The resulting FT-STS maps can be quite complex, and contain regions of high intensity. Depending on the energy, these regions can be circular, triangular, or hexagonal, as shown in Fig. 4 For example, at low energy, the dominant features in the FT-STS maps are circles centered about the corners and the center of the BZ. The contours evolve with increasing energy: their radii increase, the circles centered about the corners become triangular, while the circle in the center becomes a hexagon. Beyond the critical energy defined by the Van Hove singularity, the topology of the contours changes, and the LDOS exhibits hexagonally shaped lines of high intensity centered about the center of the BZ. With increasing energy these lines become smaller and circular again, and they disappear altogether at even higher energies.

We now review some general properties of graphite and explain our $T$-matrix calculation of the FT-STS maps in graphite in the presence of a single impurity.

Graphite has a hexagonal lattice with one valence electron per atom and two electrons per unit cell. The band structure of graphite consists of two bands with an energy dispersion approximated ${ }^{12}$ by the simple particlehole symmetric tight-binding band structure:

$$
\begin{aligned}
& \epsilon_{ \pm}(k)= \\
& = \pm \gamma \sqrt{3+2 \cos \left(\sqrt{3} k_{x} a\right)+4 \cos \left(\frac{\sqrt{3} k_{x} a}{2}\right) \cos \left(\frac{3 k_{y} a}{2}\right)}
\end{aligned}
$$

where $a$ is the distance between nearest neighbors, and $\gamma \approx 1 \mathrm{eV}$. For simplicity we will set $\gamma=1 \mathrm{eV}$ and $a=1$ in the rest of the calculation. The two bands touch at the corners of the BZ, at points $K_{0}=2 \pi / 9( \pm 2 \sqrt{3}, 0)$, $2 \pi / 9( \pm \sqrt{3}, \pm 3)$. The band structure for graphite is depicted in Fig. 1] At low energies, for wavevectors close to the corners of the BZ, the dispersion of the nodal quasiparticle excitations is linear $\epsilon_{ \pm}(k)= \pm 3 \gamma\left|k-K_{0}\right| / 2$.

The single particle density of states (DOS) of graphite can be easily obtained by integrating the spectral function, $\rho_{0}(\omega)=\int d^{2} k /\left(4 \pi^{2}\right) A(k, \omega)$, where the integral is performed over the $\mathrm{BZ}$, and

$$
A(k, \omega)=-2 \sum_{j=1,2} \operatorname{Im}\left\{\left[\omega-\epsilon_{j}(k)+i \delta\right]^{-1}\right\} .
$$

In the numerical evaluation of the single particle density of states we took a finite quasiparticle inverse lifetime $\delta=0.02 \mathrm{eV}$. The result is sketched in Fig. 2, The tiny DOS at $\omega=0$, as well as the rounding of the 


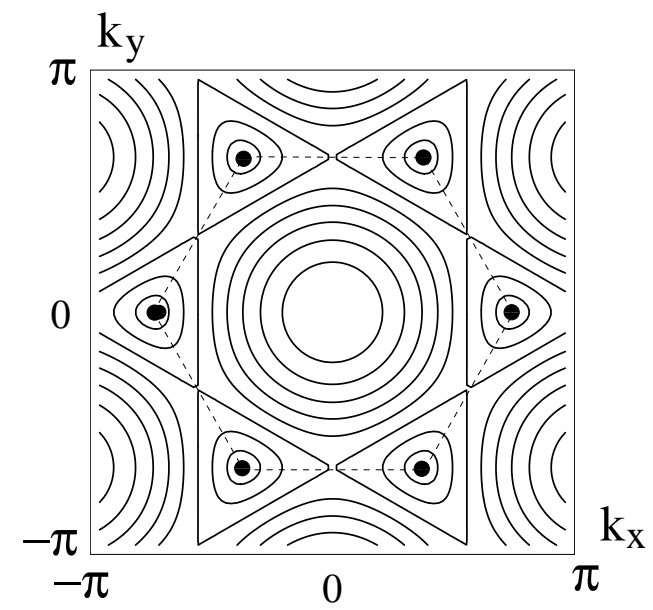

FIG. 1: The equal energy contours $\left|\epsilon_{1}\left(k_{x}, k_{y}\right)\right|=\left|\epsilon_{2}\left(k_{x}, k_{y}\right)\right|$ in graphite. The six points marked by dots correspond to the corners of the BZ (also the points with $\omega=0$ ), and the BZ is indicated by the dashed lines. The band structure at small energies consists of circular energy contours centered about the corners and the center of the BZ. At larger energies, the circles change shape and size, and above some energy $\omega=1 \mathrm{eV}$ they become hexagons and then circles centered about the center of the BZ, to disappear altogether for energies larger than $\omega=3 \mathrm{eV}$.

van Hove singularities, are due to the finite quasiparticle lifetime. When the quasiparticle inverse lifetime $\delta$ approaches zero, the density of states at the Van Hove singularity diverges $\rho_{0}\left(\omega_{V H}\right) \propto \ln (1 / \delta)$, and the density of states at zero energy goes to zero, $\rho_{0}(0) \propto \delta \ln (1 / \delta)$.

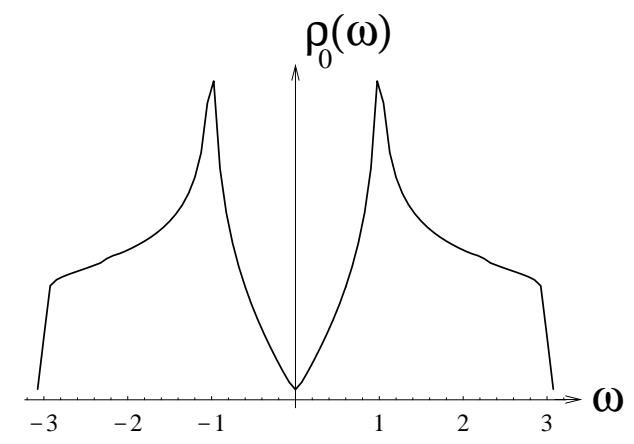

FIG. 2: The single particle density of states $\rho(\omega)$ plotted as a function of energy $\omega$. We note the v-shaped DOS of small energy and the presence of the van-Hove singularities at energies of $\pm 1 \mathrm{eV}$. At energies $|\omega| \geq 3 \mathrm{eV}$ the DOS goes to zero.

We note that the density of states is symmetric about the origin $\omega=0$ and is v-shaped for energies $|\omega|<1 \mathrm{eV}$. At the points $\omega_{V H}= \pm 1 \mathrm{eV}$, the density of states shows van Hove singularities. This is the point where equal energy contours change topology in the band structure (see
Fig. 11). As expected, the DOS goes to zero at energies $|\omega| \geq \pm 3 \mathrm{eV}$.

Following Refs. 10 and 11 we compute the effect of a single impurity on the LDOS in graphite. We define a finite temperature (imaginary time) Green's function,

$$
G\left(k_{1}, k_{2}, \tau\right)=-\operatorname{Tr} e^{-\beta(K-\Omega)} \mathrm{T}_{\tau} \psi_{k_{1}}(\tau) \psi_{k_{2}}^{+}(0),
$$

where $K=H-\mu N, e^{-\beta \Omega}=\operatorname{Tr} e^{-\beta K}$, and $\mathrm{T}_{\tau}$ is the imaginary time ordering operator. The impurity scattering problem can be solved by computing the Fourier transform of the Green's function from the $T$-matrix formulation 10,11 :

$G\left(k_{1}, k_{2}, i \omega_{n}\right)=G_{0}\left(k_{1}, i \omega_{n}\right) T\left(k_{1}, k_{2}, i \omega_{n}\right) G_{0}\left(k_{2}, i \omega_{n}\right)$,

where

$$
G_{0}\left(k, i \omega_{n}\right)=\left(\begin{array}{cc}
{\left[i \omega_{n}-\epsilon_{1}(k)\right]^{-1}} & 0 \\
0 & {\left[i \omega_{n}-\epsilon_{2}(k)\right]^{-1}}
\end{array}\right),
$$

and

$$
\begin{aligned}
& T\left(k_{1}, k_{2}, i \omega_{n}\right)=V\left(k_{1}, k_{2}\right) \\
& +\sum_{k^{\prime}} V\left(k_{1}, k^{\prime}\right) G_{0}\left(k^{\prime}, i \omega_{n}\right) T\left(k^{\prime}, k_{2}, i \omega_{n}\right) .
\end{aligned}
$$

We will assume that the impurity scattering potential is very close to a delta function so that $V$ is independent of $k$ and $k^{\prime}$,

$$
V=v\left(\begin{array}{ll}
1 & 1 \\
1 & 1
\end{array}\right)
$$

For this case we can solve Eq. (6), and obtain

$$
\begin{aligned}
T\left(i \omega_{n}\right) & =\left[I-V \bar{a} \frac{d^{2} k}{4 \pi^{2}} G_{0}\left(k, i \omega_{n}\right)\right]^{-1} V \\
& =\frac{V}{1-v \bar{a} \int \frac{d^{2} k}{4 \pi^{2}}\left[G_{0}^{11}\left(k, i \omega_{n}\right)+G_{0}^{22}\left(k, i \omega_{n}\right)\right]},
\end{aligned}
$$

where $I$ is the $2 \times 2$ identity matrix, $\bar{a}=3 \sqrt{3} / 2$ is the area of the real space unit cell of the honeycomb lattice, and the integral over $k$ is performed over the entire Brillouin zone. In the neighborhood of the impurity, spatial oscillations of the local density of states are induced. It is easier to extract information about the quasiparticle characteristics from the Fourier transform of the LDOS:

$$
\rho(q, \omega)=i \sum_{k} g(k, q, \omega)
$$

where $g(k, q, \omega) \equiv \sum_{i=1,2} G_{i i}(k, k+q, \omega)-G_{i i}^{*}(k+q, k, \omega)$, and $G(k, k+q, \omega)$ is obtained by analytical continuation $i \omega_{n} \rightarrow \omega+i \delta$ from $G\left(k, k+q, i \omega_{n}\right)$. We obtain

$$
\begin{aligned}
& \rho(q, \omega)=-2 \int \frac{d^{2} k}{4 \pi^{2}} \\
& \sum_{i=1,2} \operatorname{Im}\left\{\frac{T(\omega)}{\left[\omega-\epsilon_{i}(k)+i \delta\right]\left[\omega-\epsilon_{i}(k+q)+i \delta\right]}\right\},
\end{aligned}
$$


where $T(\omega)=v /\left\{1-v \bar{a} \int d^{2} k /\left(4 \pi^{2}\right)\left[G_{0}^{11}(k, \omega+i \delta)+\right.\right.$ $\left.\left.G_{0}^{22}(k, \omega+i \delta)\right]\right\}$. Eq.(11) is analyzed numerically and the resulting LDOS is plotted in Fig. (4) For our numerical analysis we pick values of $v=2 \mathrm{eV}$ and $\delta=0.02 \mathrm{eV}$. Also we perform our numerical integration on a $288 \times 240$ grid. We plot our results on a $144 \times 120$ grid. We have confirmed that the results have converged in the sense that changing the size of the grid leads to no observable changes in the results.

At low energies, our analysis reveals that the dominant features in the LDOS spectra are circular contours of radius $4 \omega / 3 \gamma$, centered about the corners and the center of the BZ. The shape of the contours changes with energy, and their size increases. At the energy $\omega=\omega_{V H}=1 \mathrm{eV}$, the spectral features change to hexagonal shaped contours centered about the center of the BZ. With increasing energy the contours become smaller and circular, and disappear for energies above $\omega=3 \mathrm{eV}$.

A few comments about our results are in order. We note that the intensity inside and outside the circles is not zero but finite $\frac{13}{}$, which, depending on the quasiparticle inverse lifetime $\delta$, can blur the interiors of the circles, especially at low energies.

The topology of the contours is identical for negative and positive energies. However the weight, and in some cases the sign of the peaks relative to the background, as well as the average spectral intensities are different, in particular at low energies. The asymmetry of the FTSTS spectra with respect to energy is not a direct band structure effect, since for graphite the band structure is particle-hole symmetric. It is an effect of the impurity scattering. We will discuss this asymmetry also in connection to the effect of the impurity scattering on the average density of states in Fig. [3.

For positive energies, the results presented here do not depend sensitively on the strength of the impurity potential, $v$. We have repeated our calculation for the FT-STS spectra for different values of $v$, including the limit of infinite scattering strength ("unitarity limit") and the changes are not qualitatively significant. However, at negative energies, our FT-STS pictures evolve with increasing $v$, and at large scattering strengths they become similar to their positive energy counterparts.

Fermi liquid theory is most robust at small probing energies, and at these energies we would expect the experimentally observed features to have the best agreement

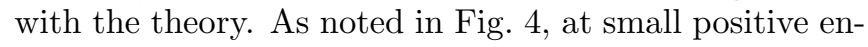
ergies the amplitude of the LDOS oscillations due to the impurity scattering is lower than at high energies, which may make the experimental observation of these oscillations more difficult. However, the fractional amplitude of the LDOS oscillations at small negative energies is larger than at higher energies.

The Van-Hove singularities which appear at high energies, are a "big" feature, so survive in some form unless there is a very serious failure of the quasiparticle picture. However, it is still possible that a strongly energy dependent quasiparticle lifetime could considerably mute the Van Hove singularities, while leaving the Fermi liquid behavior intact at much lower energies.

We note that at low energy the position of the dots/circles is determined by symmetry, while at higher energies the shape and the position of the high intensity lines is sensitive to the assumed band structure. Another interesting point is that in a two dimensional system, the general structure of singularities is lines instead of points 13 . This yields lines of high intensity regions in the in FT-STS spectra due to quasiparticle interference, as opposed to points $\frac{13}{}$.
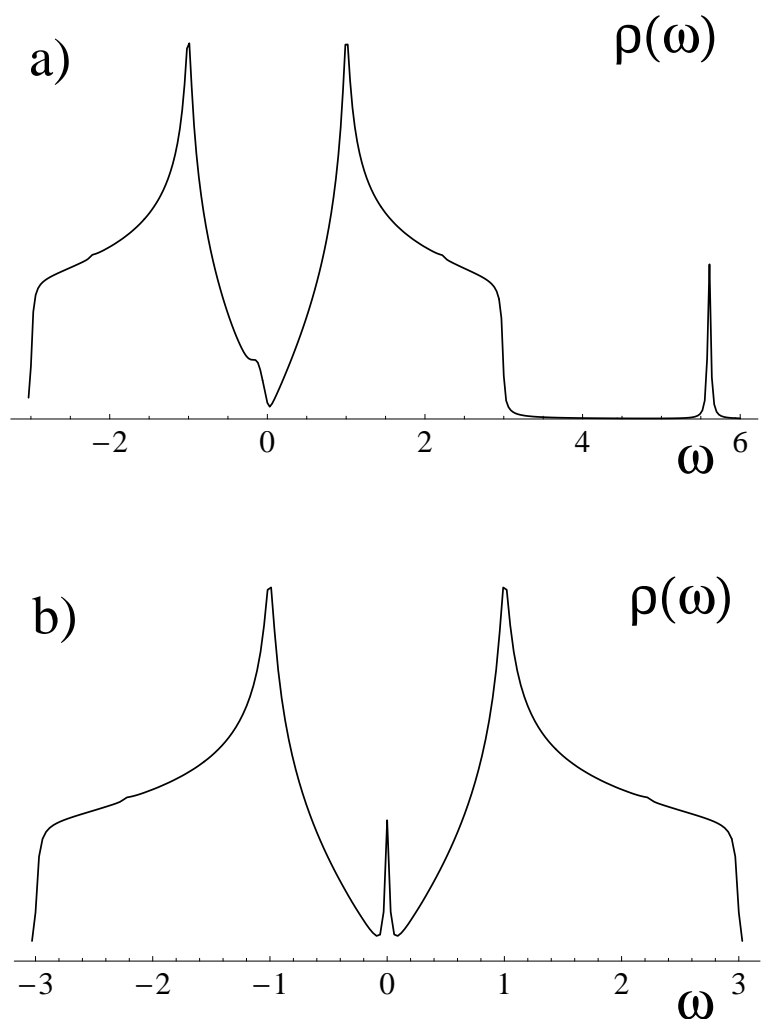

FIG. 3: The spatially averaged single particle density of states $\rho(\omega)$, in the presence of an impurity of strength a) $v=2.5 \mathrm{eV}$, and b) $v=\infty$.

Another interesting aspect we analyzed is how the spatially averaged single particle density of states $\rho(\omega)=$ $\rho_{0}(\omega)+2 c_{i m p} \rho(q=0, \omega)$ changes with the scattering strength $v$. Here $c_{i m p}$ is the concentration of impurities. We assume that $c_{i m p}$ is small, so the effects of the impurities are uncorrelated. The resulting density of states depends on the value of the impurity potential. For comparison, in Fig. 3 we give $\rho(\omega)$ for $v=2.5 \mathrm{eV}$ (a), and for $v=\infty$ (b). We fix the impurity concentration to $c=1 \%$. As expected, in the unitarity limit the effect of the impurity is to create a sharp peak centered at zero energy. For smaller impurity potential, the effect of the impurity is to create a smaller and broader peak at negative energy. One also notes the existence of a sharp anti-bound 
state at an energy larger than $3 \mathrm{eV}$. The position of the anti-bound state moves to larger and larger energies as one increases the scattering strength.

We also performed an analytical calculation of the local density of states as a function of position at low energy, where we can approximate the spectrum by a linear (Dirac) dispersion $\omega= \pm v_{F}|k|$. We focused on the case of a single impurity scattering. For points far from the impurity we found the correction to the density of states due to impurity scattering to have the form:

$$
\rho(\vec{x}, \omega)=\sum_{i=0}^{m} \frac{A_{i}}{|\vec{x}|} \cos \left(\frac{2 \omega}{v_{F}}|\vec{x}|+\vec{Q}_{i} \cdot \vec{x}+\phi_{i}\right),
$$

where $v_{F}=3 \gamma / 2$ is the Fermi velocity. Here $m=2$ is the number of the independent inter-nodal scattering vectors (that cannot be obtained from other scattering vectors by translation by a reciprocal lattice vector or by reflection). We can take, for example, $\vec{Q}_{0}=(0,0)$, $\vec{Q}_{1}=2 \pi / 9(2 \sqrt{3}, 0), \vec{Q}_{2}=2 \pi / 9(\sqrt{3}, 3)$. The coefficients $A_{i}$ and $\phi_{i}$ depend on energy and on the impurity characteristics. As expected, at large $|\vec{x}|$, the density of states decays as $1 /|\vec{x}|$. If one takes into account also the finite quasiparticle lifetime the above equation for the density of states changes to

$$
\rho(\vec{x}, \omega) \rightarrow \rho(\vec{x}, \omega) e^{-|\vec{x}| \tau / v_{F}},
$$

where $\tau=\tau(\omega)$ is the quasiparticle lifetime. A measurement of the density of states as a function of position thus reflects the position of the nodes $\left(\vec{Q}_{i}\right)$, the quasiparticle dispersion, $v_{F}$, and the energy dependence of the quasiparticle lifetime.

\section{Acknowledgments}

We would like to thank Hongwen Jiang and Jianping $\mathrm{Hu}$ for interesting discussions. C. B. has been supported by the NSF under Grant No. DMR-9985255, and also by funds from the Broida-Hirschfeller Foundation, the A. P. Sloan Foundation and the Packard Foundation. S. K. has been supported by the DOE under Grant No. DE-FG0300ER45798.
1 J. E. Hoffman, K. McElroy, D.-H. Lee, K. M Lang, H. Eisaki, S. Uchida, and J. C. Davis, Science 297, 1148 (2002).

2 C. Howald, H. Eisaki, N. Kaneko, M. Greven, and A. Kapitulnik, Phys. Rev. B 67, 014533 (2003).

${ }^{3}$ K. McElroy, R. W. Simmonds, J. E. Hoffman, D.-H. Lee, J. Orenstein, H. Eisaki, S. Uchida, J. C. Davis, Nature 422, $592(2003)$

4 A. Fang, C. Howald, N. Kaneko, M. Greven, and A. Kapitulnik, cond-mat/0404452

5 M. Vershinin, S. Misra, S. Ono, Y. Abe, Y. Ando, and A. Yazdani, Science 303, 1995 (2004).

6 J. E. Hoffman, E. W. Hudson, K. M. Lang, V. Madhavan, H. Eisaki, S. Uchida, and J. C. Davis, Science 295, 466 (2002).

7 K. McElroy, D.-H. Lee, J. E. Hoffman, K. M Lang, E. W. Hudson, H. Eisaki, S. Uchida, J. Lee, and J. C. Davis, cond-mat/0404005

8 J. Gonzales, F. Guinea, and M. A. H. Vozmediano, Phys. Rev. Lett. 77, 3589 (1996); J. Gonzales, F. Guinea, and M. A. H. Vozmediano, Phys. Rev. B 59, R2474 (1999); S.
Yu, J. Cao, C. C. Miller, D. A. Montell, R. J. D. Miller, and Y. Gao, Phys. Rev. Lett. 76, 483 (1996).

9 J. M. Byers, M. E. Flatté, and D. J. Scalapino, Phys. Rev. Lett. 71, 3363 (1993); M. I. Salkola, A. V. Balatsky, and D. J. Scalapino, Phys. Rev. Lett. 77, 1841 (1996); W. Ziegler, D. Poilblanc, R. Preuss, W. Hanke, and D. J. Scalapino, Phys. Rev. B 53, 8704 (1996); P. J. Hirschfeld, P. Wölfle, and D. Einzel, Phys. Rev. B 37, 83 (1988); A. Polkovnikov, S. Sachdev, and M. Vojta, Phys. Rev. Lett. 86, 296 (2001); D. Podolsky, E. Demler, K. Damle, and B. I. Halperin, Phys. Rev. B 67, 094514 (2003).

10 Q.-H. Wang and D.-H. Lee, Phys. Rev. B 67, 020511 (2003).

11 C. Bena, S. Chakravarty, J. Hu, and C. Nayak, Phys. Rev. B 63, 134517 (2004); C. Bena, S. Chakravarty, J. Hu, and C. Nayak, cond-mat/0405468

12 P. R. Wallace, Phys. Rev. 71, 622 (1947).

13 S. A. Kivelson, I. P. Bindloss, E. Fradkin, V. Oganesyan, J. M. Tranquada, A. Kapitulnik, and C. Howald, Rev. Mod. Phys. 75, 1201 (2003). 


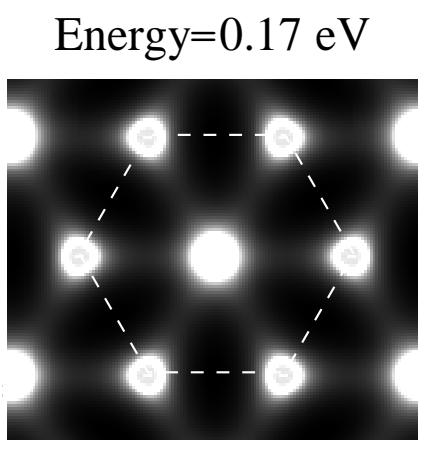

Energy $=1.0 \mathrm{eV}$

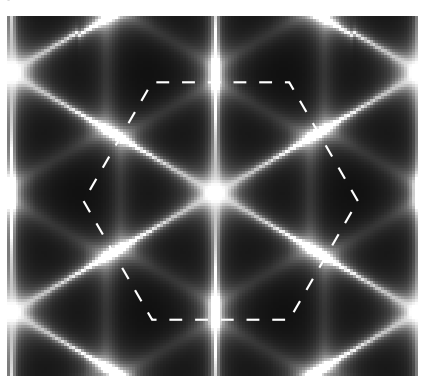

Energy $=-0.17 \mathrm{eV}$

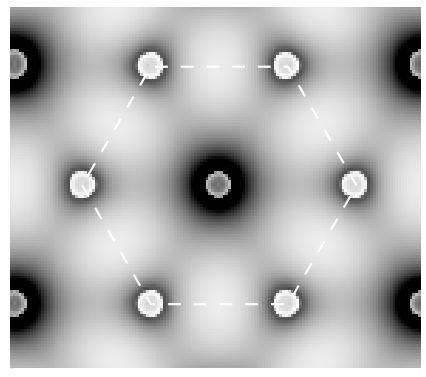

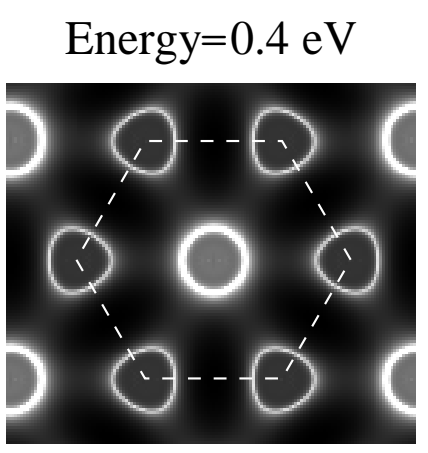

Energy $=1.1 \mathrm{eV}$

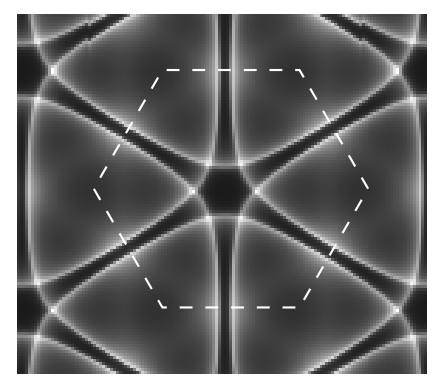

Energy $=-0.8 \mathrm{eV}$

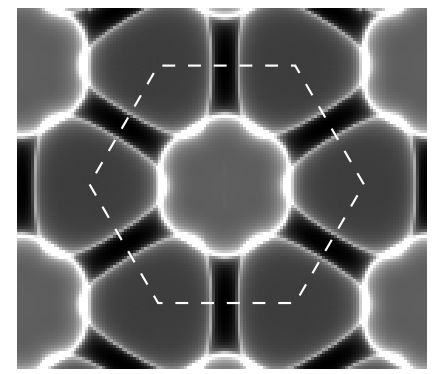

\section{Energy $=0.8 \mathrm{eV}$}

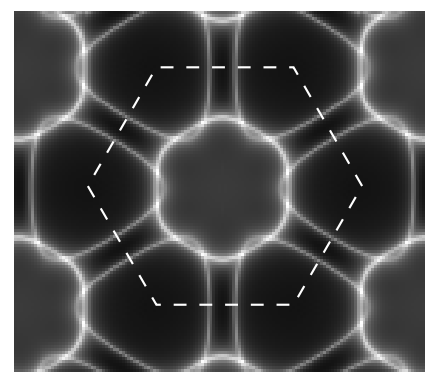

Energy $=2.0 \mathrm{eV}$

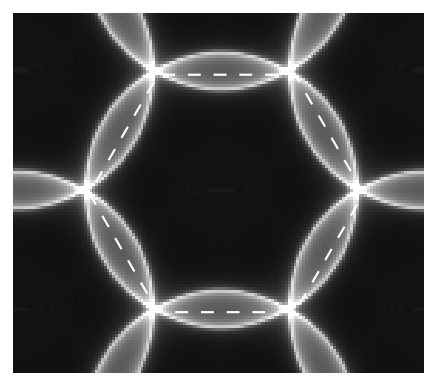

Energy $=-2.0 \mathrm{eV}$

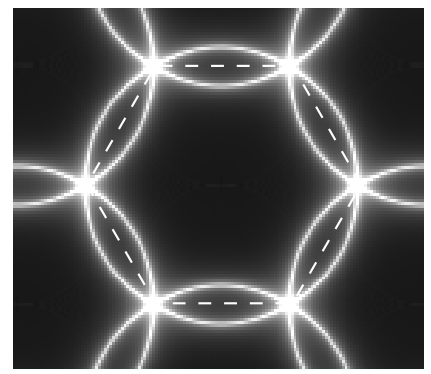

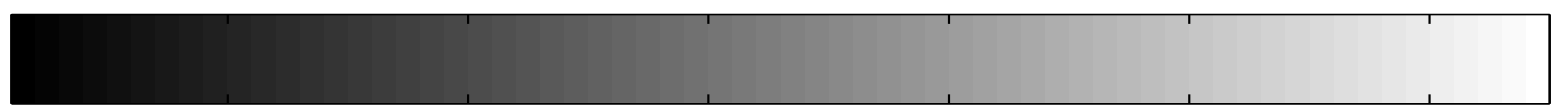

0

0.5

FIG. 4: The FT-STS quasiparticle interference maps in graphite in the presence of a single impurity. We plot $-\rho(q, \omega)$ at the energies $\omega=0.17,0.4,0.8,1,1.1,2,-0.17,-0.8$ and $-1.1 \mathrm{eV}$. The $x$ and $y$ axis of each plot are $q_{x}$ and $q_{y}$, and we display our results for $-1.15 \pi \leq q_{x} \leq 1.15 \pi$ and $-\pi \leq q_{y} \leq \pi$. For clarity we draw the BZ in dashed lines. Note the change in the contours topology with increasing energy. The plots are drawn using a normalized linear gray scale, with white being the highest (1) intensity, and black being the lowest (0) intensity, as indicated. The actual values of the FT-LDOS corresponding to the lowest (0) and highest (1) intensity are different for each energy, and we find them to be $(-0.12,1.73)$ for $0.17 \mathrm{eV},(-0.04,2.16)$ for $0.4 \mathrm{eV},(0.27,3.6)$ for $0.8 \mathrm{eV},(0.45,14.3)$ for $1.0 \mathrm{eV},(0.41,3.96)$ for $1.1 \mathrm{eV},(0.22,6.45)$ for $2.0 \mathrm{eV},(-8.2,-3.26)$ for $-0.17 \mathrm{eV}$, $(-1.1,4.07)$ for $-0.8 \mathrm{eV}$, and $(0.29,5.22)$ for $-2.0 \mathrm{eV}$ in arbitrary units 